\title{
Stochastic Non-Markovian Schroedinger equation for a three-level quantum system
}

\author{
V. Semin ${ }^{1}$, A. Pavelev ${ }^{1}$ \\ ${ }^{1}$ Samara National Research University, 34, Moskovskoye shosse, 443086, Samara, Russia
}

\begin{abstract}
Non-Markovian dynamics of a three-level system is studied with the help of stochastic Schrödinger equation (SSE). We derive a new form of SSE for a three-level system driven by four independent Ornstein-Uhlenbeck stochastic noises. The main advantage of the suggested SSE is the ensuring of the complete positivity of the reduced density operator. We demonstrate significant influence of the non-Markovian noises on the dynamics of the three-level quantum system.
\end{abstract}

Keywords: stochastic Schrödinger equation; three-level systems; non-Markovian dynamics

\section{Introduction}

Open quantum systems are usually described by the reduced density operator, which satisfies a master equation. All the master equations can be strictly divided into two classes: Markovian and non-Markovian [1]. The Markovian ones traditionally represent systems of the first order differential equations with the constant coefficients and they are well studied. In opposite, non-Markovian master equations forsake many open questions and they are intensively studied during the last several years [2]. Today it is clear that the non-Markovian master equations may be of two types either integro-differential or diferential with variable coefficients. Both types of the non-Markovian master equations are equivalent and the differential one is used more often. Unfortunately, the general form of the non-Markovian master equation, which ensures the complete positivity of the density operator is still unknown.

Another approach to describe dynamics of open quantum systems is to use Stochastic Schrödinger equation (SSE) for the wave vector driven by the noise $[3,4]$. The reduced density operator is recovered by mean of stochastic averaging over many realizations of such vectors. There is an exact correspondence between Markovian master equations and SSEs. It is obvious that the dimension of the wave vector is smaller than the dimension of the reduced density operator. This fact open a new possibilities for investigation of high-dimensional open quantum systems, such as spin chains, photosynthetic reaction centre, etc.

As in the case of non-Markovian master equations, non-Markovian SSEs are also intensively examined. The main attempts of researchers here are focused on the so called unravelling of the non-Markovian master equations [5], i.e. construction of a SSE which reproduces all the results given by the master equation. Unravelling is not always possible especially for integrodifferential master equations. On the other hand, one can generalise Markovian SSEs to non-Markovian ones without direct connection with the master equation formalism. Such an approach has many advantages and one of them is ensuring of the complete positivity of the reduced density operator.

In this paper we consider the non-Markovian generalization of the SSE for a three-level quantum system. We present results of the direct simulation of the non-Markovian SSE for this model and discuss the main difference between Markovian and non-Markovian SSEs.

\section{Model}

Three level systems can be of three different types $\Lambda, V$ and cascade. The difference between them is forbidden transitions between the energy levels. Let us consider the three-level system of $V$-types for concreteness. The Hamiltonian of the $V$ system in the photonic thermostat is [6] (we set $\hbar=1$ )

$$
H=\omega_{0} H_{1}+\Omega_{0} H_{2}+\sum_{j=1}^{\infty} \omega_{j} b_{j}^{\dagger} b_{j}+\sum_{i=1}^{\infty}\left[\left(f_{i} J_{+}+g_{i} L_{+}\right) b_{i}+\text { h.c. }\right],
$$

where $b_{j}$ and $b_{j}^{\dagger}$ are creation and annihilation operator of the $j$ th photon in the thermostate with frequency $\omega_{j}, \omega_{0} / 2$ and $\Omega_{0}+\omega_{0} / 2$ are transition frequency in the $V$ - system, $f_{i}$ and $g_{i}$ are the constant of system-photon interaction and the matrices $H_{1}, H_{2}, J_{+}, L_{+}$ are define as follow [6]

$$
H_{1}=\frac{1}{2}\left(\begin{array}{ccc}
1 & 0 & 0 \\
0 & 0 & 0 \\
0 & 0 & -1
\end{array}\right), H_{2}=\frac{1}{3}\left(\begin{array}{ccc}
1 & 0 & 0 \\
0 & 1 & 0 \\
0 & 0 & -2
\end{array}\right), J_{+}=\left(\begin{array}{ccc}
0 & 0 & 0 \\
0 & 0 & 1 \\
0 & 0 & 0
\end{array}\right), K_{+}=\left(\begin{array}{ccc}
0 & 1 & 0 \\
0 & 0 & 0 \\
0 & 0 & 0
\end{array}\right)
$$




\subsection{Markovian evolution}

The Markovian master equation for the reduced density operator can be written as [6]

$$
\frac{\partial \rho}{\partial t}=\frac{\gamma_{J}}{2}\left[\left(N_{J}+1\right)\left(2 J_{-} \rho J_{+}-J_{+} J_{-} \rho-\rho J_{+} J_{-}\right)+N_{J}\left(2 J_{+} \rho J_{-}-J_{-} J_{+} \rho-\rho J_{-} J_{+}\right)\right]+(J \leftrightarrow K),
$$

where $\gamma_{J, K}$ are the damping constants, $N_{J, K}$ are the average numbers of the heat photons on the corresponding transition.

The standard procedure of unravelling allows to write the SSE, corresponding to (3) in the following form

$$
d|\psi\rangle=-\frac{\gamma_{J}}{2}\left(\left(N_{J}+1\right) J_{+} J_{-}+N_{J} J_{-} J_{+}\right)|\psi\rangle d t+i \sqrt{\gamma_{J}\left(N_{J}+1\right)} J_{-}|\psi\rangle d W_{J}^{1}+i \sqrt{\gamma_{J} N_{J}} J_{+}|\psi\rangle d W_{J}^{2}+(J \leftrightarrow K),
$$

where $W_{J, K}^{1,2}$ are independent standard Wiener processes. It is easy to verify using Ito calculus that $\rho=\mathbb{E}(|\psi\rangle\langle\psi|)$ satisfies the master equation (3).

\subsection{Non-Markovian evolution}

To describe non-Markovian effects Barchielli in [7] suggested to replace Markovian Wiener processes in (4) by some nonMarkovian noises. One of the simplest non-Markovian noises is the Ornstein-Uhlenbeck one, which is satisfies the stochastic equation

$$
d X=-k X d t+d W
$$

where $k>0$ is some constant. By substitution the Ornstein-Uhlenbeck processes (5) instead of the Wiener increments in (4) we derived the following non-Markovian SSE

$$
\begin{aligned}
d|\tilde{\psi}\rangle=-\left(\frac{\gamma_{J}}{2}\left(N_{J}+1\right) J_{+} J_{-}+\right. & \left.\frac{\gamma_{J}}{2} N_{J} J_{-} J_{+}+i k_{J}^{1} X_{J}^{1} \sqrt{\gamma_{J}\left(N_{J}+1\right)} J_{-}+i k_{J}^{2} X_{J}^{2} \sqrt{\gamma_{J} N_{J}} J_{+}\right)|\tilde{\psi}\rangle d t \\
& +i \sqrt{\gamma_{J}\left(N_{J}+1\right)} J_{-}|\tilde{\psi}\rangle d W_{J}^{1}+i \sqrt{\gamma_{J} N_{J}} J_{+}|\tilde{\psi}\rangle d W_{J}^{2}+(J \leftrightarrow K) .
\end{aligned}
$$

Unfortunately, the above SSE is not a mean-1 martingale and we have to somehow modify the equation to satisfy the martingale property. It is straightforward to check that to be a mean-1 martingale Eq. (7) needs 4 more terms in the drift part, namely

$$
\begin{array}{r}
d|\tilde{\psi}\rangle=-\left(\frac{\gamma_{J}}{2}\left(N_{J}+1\right) J_{+} J_{-}+\frac{\gamma_{J}}{2} N_{J} J_{-} J_{+}+i k_{J}^{1} X_{J}^{1} \sqrt{\gamma_{J}\left(N_{J}+1\right)}\left(J_{-}+J_{+}\right)+i k_{J}^{2} X_{J}^{2} \sqrt{\gamma_{J} N_{J}}\left(J_{-}+J_{+}\right)\right)|\tilde{\psi}\rangle d t \\
+i \sqrt{\gamma_{J}\left(N_{J}+1\right)} J_{-}|\tilde{\psi}\rangle d W_{J}^{1}+i \sqrt{\gamma_{J} N_{J}} J_{+}|\tilde{\psi}\rangle d W_{J}^{2}+(J \leftrightarrow K) .
\end{array}
$$

Obviously, that Eq. (7) is transformed to Eq. (4) when all $k_{m}^{l}=0$. Moreover, it is easy to prove that Eq. (7) is a martingale, i.e. $\mathbb{E}(\langle\tilde{\psi}(t) \mid \tilde{\psi}(t)\rangle)=1$ and $\tilde{\rho}(t)=\mathbb{E}(|\tilde{\psi}(t)\rangle\langle\tilde{\psi}(t)|)$ is a completely positive operator by construction. Note, that operator $\tilde{\rho}(t)$ does not satisfy the Markovian master equation (3) and, even more, we cannot construct any closed master equation for this operator due to the presence of the noise terms in the drift part of the equation (7). All the above mentioned facts demonstrate the uniqueness of Eq. (7).

\section{Results of simulation}

The Non-Markovian SSE (7) can be efficiently simulated. It is possible to do if we add to the three components of the wave vector $|\tilde{\psi}\rangle$, four stochastic equations for the Ornstein-Uhlenbeck noises. Resulting seven equations form a closed system and may be numerically solved by any suitable algorithm. The initial values for the Ornstein-Uhlenbeck processes are normally distributed random numbers with zero mean and unit standard deviation.

In this paper we use the Euler stochastic algorithm [8], which for our problem can be written in the following general form

$$
\Psi_{n+1}=A \Psi_{n} \Delta t+\sum_{i=1}^{4} B_{i} \Psi_{n} \delta W_{i},
$$

where $A, B_{i}$ are constant matrices, $\Delta t$ is the time step, $\delta W_{i}$ is independent normal distributed variables $N(0, \Delta t)$. The vector $\Psi=\left(\psi_{x}, \psi_{y}, \psi_{z}, X_{J}^{1}, X_{J}^{2}, X_{K}^{1}, X_{K}^{2}\right)^{T}$ has seven components. Initial conditions is $\Psi_{0}=(1,0,0, N(0,1), N(0,1)$, $N(0,1), N(0,1))^{T}$.

The results of simulation are presented in Fig. 1. The results was averaged over $10^{4}$ realizations. The error bars are also included in the graphic. In the same pictures we draw the dynamics given by the Markovian master equation (3). One can see that the non-Markovian noise has significant effect on dynamics and cannot be neglected in general. 


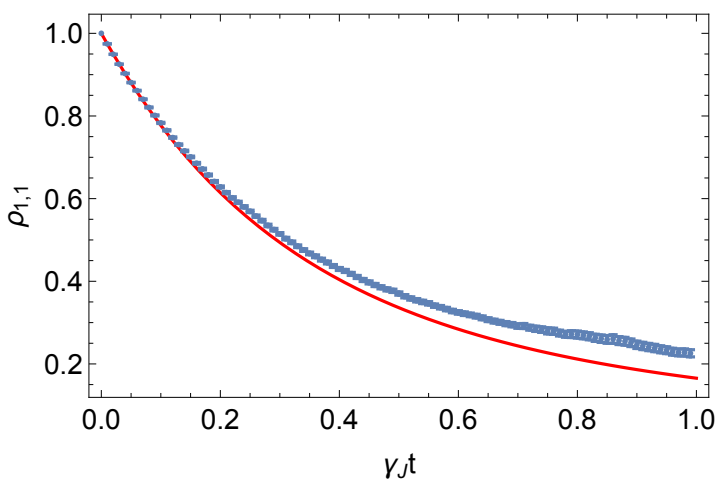

Fig.1. Evolution of the upper state. Red curve is the Markovian dynamics and blue dots are the non-Markovian dynamics. Parameters: $\gamma_{K}=2 \gamma_{J}, N_{J}=0.2$, $N_{K}=0.3, k_{J}^{1}=k_{K}^{1}=0.3, k_{J}^{2}=k_{K}^{2}=0.5$.

\section{Conclusion}

In this paper we have derived the non-Markovian SSE for a three-level quantum system driven by the four independent Ornstein-Uhlenbeck processes. The SSE has unique properties which are hard to achieve in other approaches to non-Markovian dynamics. Especially, it is the complete positivity of the density operator $\tilde{\rho}(t)=\mathbb{E}(|\tilde{\psi}(t)\rangle\langle\tilde{\psi}(t)|)$ for all time. The suggested SSE can be efficiently simulated using any appropriate algorithm and due to stochastic nature of the equation the solution can be easy parallelized to perform calculation on a supercomputer or GPU.

It is shonw that quantum dynamics given by the non-Markovian SSE is significantly differ from Markovian one and this fact should be taken into account in the explanation of future experiments with quantum ensembles.

The general features of SSE described in this paper for the three-level systems are valid for arbitrary quantum systems. Moreover, the SSE has dimension much smaller than the corresponding master equation. This means that using SSE technique one can describe high dimensional quantum systems. This may be relevant for understanding biological phenomena, such a photosyntheses.

\section{References}

[1] Breuer HP, Petruccione F. The theory of open quantum systems. Oxford University Press: Oxford, 2002.

[2] Breuer HP, Laine EM, Piilo J, Vacchini B. Non-Markovian dynamics in open quantum systems. Rev. Mod. Phys. 2015; 88: P. 021002.

[3] Barchielli A, Gregoratti M. Quantum Trajectories and Measurements in Continuous Time. Springer: Berlin, 2009

[4] Semina I, Semin V, Petruccione F, Barchielli A. Stochastic Schrdinger Equations for Markovian and non-Markovian cases. Open Sys. Inf. Dyn. $2014.21: 1440008$.

[5] Barchielli A, Pellegrini C. Jump-diffusion unravelling of a non Markovian generalized Lindblad master equation. J. Math. Phys. 2010. 51: 112104

[6] Mikhailov VA, Troshkin NV. Relaxation of a three-level atom interacting with a thermostat and an external stochastic field. Proc. of SPIE 2015. $9917: 991731$

[7] Barchielli A, Pellegrini C, Petruccione F. Quantum trajectories: memory and continuous observation. Phys. Rev. A. 2012. 86: 063814

[8] Platen E, Bruti-Liberati N. Numerical Solution of Stochastic Differential Equations with Jumps in Finance. Springer-Verlag: Berlin, 2010. 